\title{
Production and Isolation of Magnetic Protein Crystals in HEK293T Cells
}

Thomas L. Li and Bianxiao Cui*

Department of Chemistry, Stanford University, Stanford, California, USA

*For correspondence: bcui@stanford.edu

\begin{abstract}
[Abstract] Advances in protein engineering have enabled the production of self-assembled protein crystals within living cells. Our recent publication demonstrates the production of ftn-PAK4, which is a ferritin-containing crystal that can mineralize iron and become magnetic when isolated. We have developed an optimized protocol for the production and isolation of PAK4-based crystals. The crystals are first grown in low-passage HEK293T cells, released using a lysis buffer containing NP-40 and DNase, and collected under careful centrifugation conditions. Our protocol maximizes the purity and yield of crystals and is quick and straightforward.
\end{abstract}

Keywords: Protein Crystal, Isolation, ftn-PAK4, “In cellulo", Crystal

[Background] Recent works have reported the production and isolation of "in cellulo" crystals through the heterologous expression of proteins in living cells. These crystals have varied applications, such as cargo delivery (ljiri et al., 2009) or x-ray structure determination (Baskaran and Ang, 2015). The properties of the crystals vary, but they are generally quite large relative to the cell, ranging from 1-2 $\mu \mathrm{m}$ up to hundreds of $\mu \mathrm{m}$ in size (Schönherr et al., 2015). In our recent work, we modified inka-PAK4 crystals to create ftn-PAK4, which is a ferritin-containing crystal that can mineralize enough iron to be attracted to a nearby permanent magnet (Li et al., 2019).

The production and isolation of intact protein crystals poses several unique experimental challenges. Because the crystals are so large, purification methods such as gel electrophoresis cannot be used. Likewise, because the crystals are protein-based, harsher lysis conditions such as SDS will disassemble the crystals. However, clean suspensions can be critical for downstream applications. For example, excessive debris in an inka-PAK4 suspension can trap auto-oxidized iron and stick to crystals, generating spurious magnetic attraction results.

Here, we present a protocol for the production, isolation, and iron loading of ftn-PAK4 and inka-PAK4 crystals. It is possible to achieve results using only deionized water as the lysis buffer, but we present several optimizations that significantly improve crystal yield and minimize unwanted debris. These considerations should inform future work on other protein crystals, both for their production and isolation as well as their functional applications. 


\section{Materials and Reagents}

1. Pipettes and Pipette Tips

2. $1.5 \mathrm{ml}$ Eppendorf centrifuge tubes

3. Glass slides

4. 6 Well Tissue Culture Plate, Tissue Culture Treated, Flat Bottom (Fablab, catalog number: FL7105)

5. HEK 293T cells (< 10 passages) (ATCC, catalog number: CRL-3216)

6. DMEM High Glucose without Sodium Pyruvate (Gibco, catalog number: 11965092)

7. Penicillin/Streptomycin (Gibco, catalog number: 15140122)

8. Heat Inactivated Fetal Bovine Serum (Gibco, catalog number: 10438034)

9. Lipofectamine 2000 (Invitrogen, catalog number: 11668030)

10. Opti-MEM Reduced Serum Medium (Gibco, catalog number: 31985070 )

11. GFP-PAK4, ftn-PAK4, and inka-PAK4 plasmids

12. HEPES Buffer, $1 \mathrm{M}, \mathrm{pH}$ 7.2-7.5 (Gibco, catalog number: 15630130)

13. NP-40 (Sigma, catalog number: 18896 , also known as Igepal CA-630)

14. Deoxyribonuclease I (DNase) (Worthington, catalog number: LS002007)

15. Earle's Balanced Salt Solution (EBSS) (Life Technologies, catalog number: 14155-063)

16. Ferrous Ammonium Sulfate Hexahydrate (FAS) (Sigma-Aldritch, catalog number: FX0245)

17. D-Mannitol (Sigma-Aldrich, catalog number: M4125)

18. Concentrated Hydrochloric Acid (Fisher Scientific, catalog number: A144-500)

19. Potassium Ferrocyanide Trihydrate (MP Biomedicals, catalog number: 0215256080)

20. Lysis buffer (see Recipes)

21. DNase stock solution (see Recipes)

22. Iron loading stock solution (see Recipes)

23. Prussian Blue stain (see Recipes)

\section{Equipment}

1. Centrifuge (Eppendorf, model: 5804)

2. Rocker (VWR Scientific Rocking Platform, Model 100)

3. Microscope with Brightfield and Fluorescence Imaging (Leica, model: DMI 6000B)

\section{Procedure}

A. Transfection

1. Prepare cells

Plate HEK293T cells at approximately $60 \%$ confluency in a 6-well culture plate. Ensure that the cells have not been passaged more than 10 times before transfection. 
Please cite this article as: Li and Cui, (2020). Production and Isolation of Magnetic Protein Crystals in HEK293T Cells,Bio-protocol 10 (14): e3684. DOI:

Note: Cells can be plated any time before transfection, including immediately beforehand, as long as the density is $60 \%$ at the time of transfection.

2. Transfection

Transfect HEK293T cells using Lipofectamine 2000 according to the manufacturer's instructions.

a. Use $2 \mu \mathrm{g}$ of total plasmid, and $6 \mu \mathrm{l}$ of Lipofectamine 2000 per well, for a 1:3 plasmid to reagent ratio.

b. To produce ftn-PAK4, use $1 \mu \mathrm{g}$ of ftn-PAK4 plasmid and $1 \mu \mathrm{g}$ of inka-PAK4 plasmid. To produce inka-PAK4, use $2 \mu \mathrm{g}$ of inka-PAK4 plasmid. The plasmid concentration is not important, only the total mass of DNA.

c. To aid in crystal visualization through fluorescence microscopy, include $0.2 \mu \mathrm{g}$ GFP-PAK4 plasmid in the plasmid mix. It is not necessary to increase the amount of Lipofectamine used.

3. Wait for crystal growth

The first crystals should appear $24 \mathrm{~h}$ after transfection. While inka-PAK4 crystals should be immediately visible, ftn-PAK4 crystals may not be obvious after $24 \mathrm{~h}$. To maximize crystal yield and size, maintain cells in culture for $72 \mathrm{~h}$ after transfection before isolation (Figure 1).

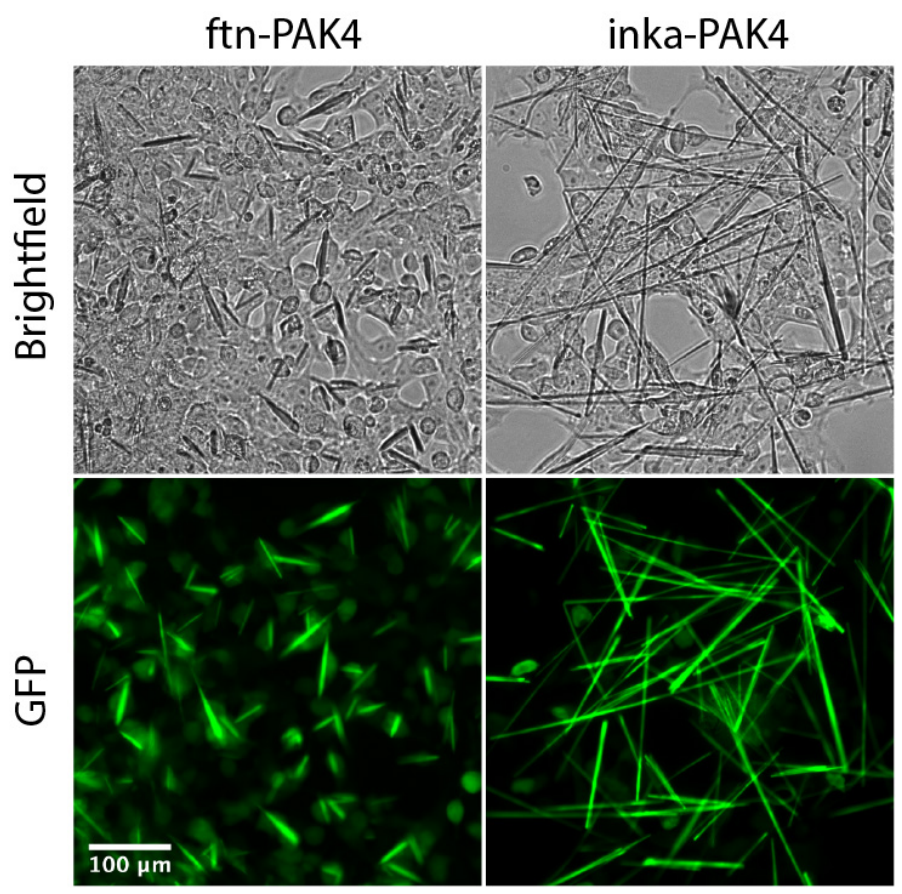

Figure 1. HEK293T cells containing ftn-PAK4 and inka-PAK4 crystals, with GFP-PAK4, 72 $\mathrm{h}$ after transfection. These crystals are ready for isolation. 


\section{B. PAK4 isolation}

1. Lyse Cells

Remove the media from the cells, and gently rinse once with $1 \mathrm{ml}$ room-temperature sterile deionized water. Add $500 \mu \mathrm{l} \mathrm{lysis} \mathrm{buffer} \mathrm{and} 10 \mu \mathrm{l}$ DNase solution directly to the cell culture well, and place on a rocker at $50 \mathrm{tilts} / \mathrm{min}$ at room temperature for $40 \mathrm{~min}$.

The presence of DNase in the lysis buffer is critical to high yields. Without DNase, a sticky pellet will form from the lysed cells' DNA, which will trap most of the crystals (Figure 2).

Without DNAse

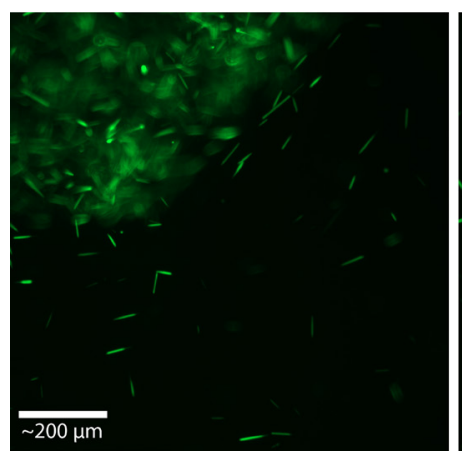

With DNAse

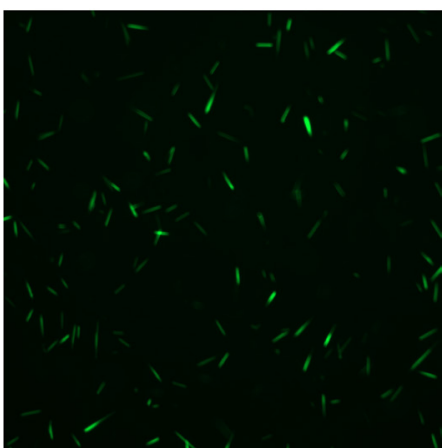

Figure 2. A ftn-PAK4 isolation without, and with DNase. In the DNase-free condition, many of the crystals have been trapped in a sticky pellet, substantially reducing the concentration of free crystals.

2. Centrifuge

After rocking, transfer the lysed cell solution to $1.5 \mathrm{ml}$ Eppendorf centrifuge tubes. Centrifuge at $400 \mathrm{rcf}$ for $1 \mathrm{~min}$ in a swinging-bucket centrifuge. Discard the supernatant and resuspend the pellet in $500 \mu \mathrm{l}$ of $0.1 \mathrm{M}$ HEPES buffer.

Note: The supernatant will still contain crystals, which can be collected using a second centrifugation.

3. Verify Isolation

Check crystal yield by pipetting a few $\mu \mathrm{l}$ of the suspension onto a coverslip and looking at it under the microscope. Isolated crystals should be visible through the eyepiece using a 10x objective under both brightfield and GFP illuminations.

C. Iron loading and assay

1. Add Iron

Prepare a solution of FAS (50 mM, 100x) and mannitol (500 mM, 100x) in sterile deionized water. Add $1 \mu \mathrm{l}$ of each stock solution to a $100 \mu \mathrm{l}$ aliquot of the crystal suspension. Incubate at room temperature for exactly $15 \mathrm{~min}$.

Notes:

a. The final concentrations are $500 \mu \mathrm{M}$ FAS and $5 \mathrm{mM}$ mannitol. The mannitol reduces the autooxidation of the iron. 
b. The FAS stock solution must be made immediately before use, as the $\mathrm{Fe}^{2+}$ will rapidly oxidize to the insoluble $\mathrm{Fe}^{3+}$.

c. The final iron-loaded suspension may be a light yellow/orange color. The darker this color is, the more $\mathrm{Fe}^{3+}$ precipitate there is.

2. Wash Iron

Centrifuge samples at $400 \mathrm{rcf}$ in a swinging bucket centrifuge for $1 \mathrm{~min}$. Discard the supernatant and resuspend the pellet in $100 \mu \mathrm{l}$ of HEPES buffer.

Notes:

a. It is important to match the volume of the wash buffer with the original suspension volume.

If a larger volume, like $500 \mu l$, is used, the ftn-PAK4 suspension may not pellet during centrifugation, though the WT-PAK4 will.

b. Common failure: If the resulting pellet is a yellow/orange color, the iron incubation step was likely too long, and analysis will be confounded by the presence of $\mathrm{Fe}^{3+}$ precipitates.

3. Prepare Prussian Blue (PB) Stain

Prepare a solution of $5 \%$ concentrated $\mathrm{HCl} v / \mathrm{v}$ and $1 \%$ potassium ferrocyanide $\mathrm{w} / \mathrm{v}$ in water.

Note: It is important to prepare the PB stain immediately before use, because $\mathrm{Fe}^{2+}$ will oxidize and create Prussian Blue over time. While this oxidized solution will still work for approximately $48 \mathrm{~h}$, it will contain a significant suspension of Prussian Blue particles.

4. Add PB Stain

To verify iron loading, pipet a $10 \mu \mathrm{l}$ droplet of iron-loaded PAK4 crystals onto a glass slide or coverslip and add $1 \mu \mathrm{l}$ of the PB stain. The droplet can be immediately checked under brightfield optical microscopy (Figure 3).

Note: If iron loading is successful, ftn-PAK4 crystals should have a deep blue color in the center. Inka-PAK4 crystals may be a faint blue, depending on the optics of the microscope.

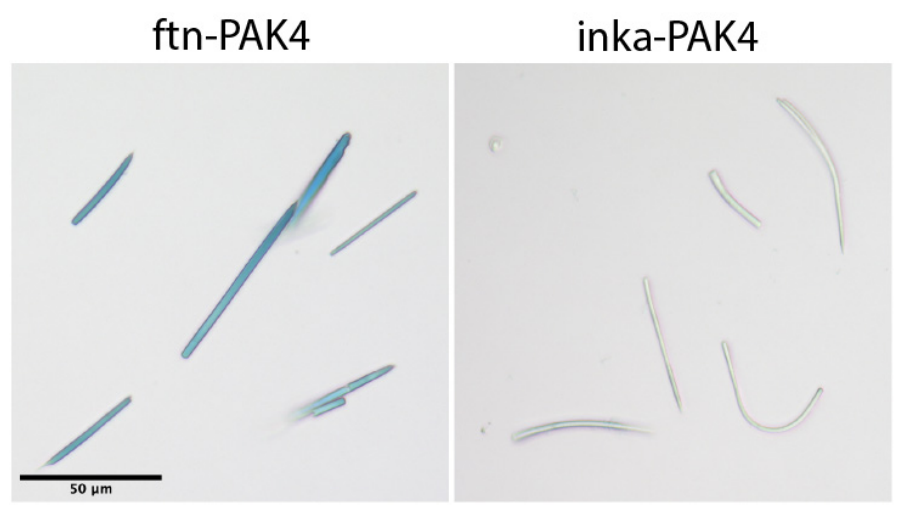

Figure 3. Ideal colors for ftn-PAK4 and inka-PAK4 crystals after iron loading and PB stain 


\section{$\underline{\text { Notes }}$}

1. The passage number of the cells used is a critical variable in crystal yield and purity. Highpassage cells will grow fewer, smaller crystals, and crystal suspensions from high-passage cells will contain significantly more debris.

2. After iron loading, if the mannitol is not removed from the solution before PB stain, it will react with the PB stain to create a precipitate over several min. This precipitate doesn't interfere with staining as a brief check, but it will interfere with clean imaging.

3. When imaging the PB stained crystals, it is important to use brightfield microscopy and not phase contrast, as phase contrast will distort colors.

4. Some variables are more important than others.

a. The following variables are highly sensitive to variation: cell passage number, centrifugation speed and time, iron loading stock concentrations, and iron loading duration.

b. The following variables are less sensitive to variation: Lysis duration and rocking speed, and PB stain concentrations and duration.

c. While the presence of mannitol helps to obtain cleaner results, its presence is not necessary to achieve iron loading of ftn-PAK4.

\section{$\underline{\text { Recipes }}$}

1. Lysis buffer

0.1 M HEPES Buffer

$1 \% \mathrm{v} / \mathrm{v} \mathrm{NP}-40$

Store at room temperature

2. DNase stock solution

12,500 Units/ml

Dissolved in EBSS

Store at $-20{ }^{\circ} \mathrm{C}$

3. Iron loading stock solution

$1 \mathrm{ml}$ MilliQ water

19.6 mg Ferrous Ammonium Sulfate (500 mM stock)

$91 \mathrm{mg}$ Mannitol (500 mM stock)

Note: Do not store-make fresh each time.

4. Prussian Blue stain

$1 \mathrm{ml}$ MilliQ water

$50 \mu \mathrm{l}$ Concentrated Hydrochloric Acid

$5 \mathrm{mg}$ Potassium Ferrocyanide

Note: Do not store-make fresh each time. 


\section{Acknowledgments}

Protocol was based on "Engineering a Genetically Encoded Magnetic Protein Crystal", in Nano Letters 2019 (Li et al., 2019). This work was supported Stanford Interdisciplinary Graduate Fellowship in association with the Wu Tsai Neurosciences Institute (T.L.L.), Packard Fellowship and Engineering (B.C.), and NIH 1R01GM125737 (B.C.).

\section{Competing interests}

The authors declare that there are no competing interests or conflicts of interest.

\section{References}

1. Baskaran, Y. and Ang, K. C. (2015). An in cellulo-derived structure of PAK4 in complex with its inhibitor Inka1. Nat Commun 6: 8681.

2. Ijiri, H., Coulibaly, F., Nishimura, G., Nakai, D., Chiu, E., Takenaka, C., Ikeda, K., Nakazawa, H., Hamada, N., Kotani, E., Metcalf, P., Kawamata, S. and Mori, H. (2009). Structure-based targeting of bioactive proteins into cypovirus polyhedra and application to immobilized cytokines for mammalian cell culture. Biomaterials 30(26): 4297-4308.

3. Li, T. L., Wang, Z., You, H., Ong, Q., Varanasi, V. J., Dong, M., Lu, B., Pasca, S. P. and Cui, B. (2019). Engineering a Genetically Encoded Magnetic Protein Crystal. Nano Lett 19(10): 69556963.

4. Schönherr, R., Klinge, M., Rudolph, J. M., Fita, K., Rehders, D., Lubber, F., Schneegans, S., Majoul, I. V., Duszenko, M., Betzel, C., Brandariz-Nunez, A., Martinez-Costas, J., Duden, R. and Redecke, L. (2015). Real-time investigation of dynamic protein crystallization in living cells. Struct Dyn 2(4): 041712. 